\title{
Das Deutsche Wörterbuch von Jacob Grimm und Wilhelm Grimm als Nationaldenkmal
}

1. Nationales Symbol oder wissenschaftlicher Meilenstein?

2. Die Idee des Nationalwörterbuchs zu Beginn des 19. Jahrhunderts und die Konzeption des Grimmschen Wörterbuchs

3. National-symbolische Merkmale des Deutschen Wörterbuchs

3.1. Die Quellen- und Stichwortwahl

3.2. Die Belegpräsentation

3.3. Vorrang von Etymologie und Formgeschichte vor Semantik

4. Nicht-nationale Merkmale des Deutschen Wörterbuchs

5. Resümee

6. Literatur

\section{Nationales Symbol oder wissenschaftlicher Meilenstein?}

Deutsche geliebte landsleute, welches reichs, welches glaubens ihr seiet, tretet ein in die euch allen aufgethane halle eurer angestammten, uralten sprache, lernet und heiliget sie und haltet an ihr, eure volkskraft und dauer hängt in ihr. noch reicht sie über den Rhein in das Elsasz bis nach Lothringen, über die Eider tief in Schleswigholstein, am ostseegestade hin nach Riga und Reval, jenseits der Karpathen in Siebenbürgens altdakisches gebiet. Auch zu Euch, ihr ausgewanderten Deutschen, über das salzige meer gelangen wird das buch und euch wehmütige, liebliche gedanken an die heimatsprache eingeben oder befestigen, mit der ihr zugleich unsere und euere dichter hinüber zieht, wie die englischen und spanischen in Amerika fortleben.

Mit diesen Worten beendete Jacob Grimm am 2. März 1854 seine Vorrede zum ersten und damit nach zwei Jahren des lieferungsweisen Erscheinens abgeschlossenen Band des Deutschen Wörterbuchs (abgekürzt DWB). Die Imaginierung des Werks als „aufgethane halle“ der „angestammten, uralten sprache" mag die Zeitgenossen an die zwölf Jahre zuvor eröffnete Walhalla bei Regensburg erinnert haben. Grimm stellte das Wörterbuch damit ganz explizit in die Reihe national-politischer Symbole, die mit dem Leipziger Völkerschlachtdenkmal begann und noch nach der Reichseinigung in zahllosen Bismarckstatuen und -türmen ihre Fortsetzung fand. Denkmäler aus 
Stein wie die etwa von Josef Görres angestellten Pläne zur Vollendung des Kölner Doms und der Bau des Germanischen Nationalmuseums in Nürnberg (gegründet 1852) gehören ebenso in diese Reihe hinein wie einige Denkmäler aus Papier: die Sammlung deutscher historischer Quellenschriften in Form der monumenta Germaniae historica ab 1826 nach den Plänen des Freiherrn vom Stein (vgl. Sellin 1988) und auch die Sammlung des historischen deutschen Sprachschatzes im Gefäß eines Nationalwörterbuchs.

Die Topoi, mittels derer das Deutsche Wörterbuch als nationales Symbol bezeichnet und in dieser Funktion tradiert wurde, reichen von den allerersten öffentlichen Reaktionen ab Mai 1852 bis in die 80er Jahre unseres Jahrhunderts, in denen das Werk als Taschenbuch nachgedruckt wurde. Da war in chronologischer Reihenfolge die Rede vom vaterländischen „Denkmal“, vom „Schatz“ und der „reichhaltige(n) Fundgrube“; Grimm selbst nennt das Wörterbuch in der Vorrede ein „nachhaltige(s) füllhorn“ (DWB 1, XIII). Eine Reaktion Alexander von Humboldts vom Juni 1852 auf die 1. Lieferung zeigt, wie sehr der Aspekt der nationalen Symbolik im Vordergrund stand:

Wo sollte ich die Worte hernehmen, um Ihnen und Ihrem mir so theuren Bruder zu danken für das großartige Werk, das unser zerspaltenes und geistig unzuverödendes Vaterland Ihnen beiden verdankt. Wenn man in trüber Erinnerung an das denkt, was wir 1848 u. 49 hoffen konnten, so empfindet man die erste Freude wieder durch Ihr Werk, durch Ihre Gabe. Grösseres hat kein Volk. Ihr Buch ist dazu ein Geschichtsbuch, ein Denkmal der organischen Entwicklung von Sprache und Volksleben, den indischen, persischen, gothischen Osten mit dem Romanischen, Keltischen verbindend, das einzige Wörterbuch, das man lesen kann wie ein Geschichtsbuch. ${ }^{1}$

Wird die Nation hier in der Sprache als Geschichtsquelle und somit im Kulturellen verankert, so kann nach 1871 der Bezug zum Politischen unmittelbar hergestellt werden. Typisch hierfür ist Rudolf Hildebrands Bild vom „reichste(n) rechte(n) futterspeicher“ des „hengest(es)“ Deutschland, der seinen Reiter in Bismarck endlich gefunden habe (DWB 11, If.). Der DWB-Bearbeiter Theodor Kochs mahnte 1939/40 die Vollendung des Werks als „Ehrenpflicht der Nation“ an (Kirkness/Kühn/Wiegand 1991, XXf.). In der Gegenwart schließlich tritt an die Stelle der nationalen eine (konsum-)gesellschaftliche Funktion; Walter Jens nennt das DWB 1984 „das Vorratshaus der Deutschen“, „ein Schatzhaus“, „ein(en) Bazar, ein gewaltiges Warenhaus des Geistes“, „ein unerschöpfliches Angebot" (Jens 1984, 29).

E negativo wurde die nationale Funktion des DWB auch durch die Kritik bestätigt, die in der Lexikographiegeschichte tendenziell marginalisiert wurde: Bei den Kritikern der Anfangsphase, Sanders und Wurm, ging es nicht um irgendein Nachschlagewerk, sondern um die „richtige“ Realisie-

Brief an Jacob Grimm. Aus der Handschrift zit. bei Hartmut Schmidt 1986, 713. 
rung der von allen getragenen Idee des Nationalwörterbuchs. ${ }^{2}$ Nach der Fertigstellung 1960 warf der Journalist Walther Boehlich dem DWB Rechtslastigkeit und Nationalismus vor. ${ }^{3}$ Paradoxerweise wurden Jacob Grimm, die Germanistik und das DWB als ihr Repräsentant etwa zur selben Zeit in der DDR für die bürgerlich-antifeudale und freiheitlich-revolutionäre Tradition Deutschlands in Beschlag genommen. ${ }^{4}$ Auch hierfür stellt Jacob Grimm in seiner Wörterbuch-Vorrede und anderswo geeignete Belege zur Verfügung: Im ersten Abschnitt der Vorrede erinnerte er die Öffentlichkeit daran, dass er und sein Bruder zu den sieben Göttinger Professoren gehörten, die wegen ihrer Verfassungstreue 1837 ihrer Ämter enthoben wurden ${ }^{5}$; Grimm führte die Entstehung des Wörterbuchs geradezu auf diese Amtsenthebung und die ihr zugrundeliegenden vaterländischen Motive zurück:

mag das werk, dessen beginn auf des geliebten vaterlandes altar wir nun darbringen, einst vollführt gegründetere zuversicht erwecken, dasz es im andenken der nachwelt haften und nicht schwinden werde, so ist uns damit alles leid vergolten. (DWB 1, II)

Dass das Deutsche Wörterbuch von Jacob Grimm und Wilhelm Grimm von Anfang an eine national-symbolische Funktion besaß und in transformierter Form sogar bis in die Gegenwart hinein behielt, ist, wie die kritischen und die zustimmenden Kommentierungen ebenso zeigen wie die diversen ideologischen Etikettierungen, unbezweifelbar und mehrfach ausführlich belegt worden. ${ }^{6}$ Demgegenüber steht allerdings eine ebenfalls imposante Reihe von Belegen, die das Deutsche Wörterbuch als ein äuBerst unpopuläres und wissenschaftlich-hermetisches Werk, obendrein als eine mehr suchende Abhandlung und Erörterung denn als ergebnisorientierte Wissensdarstellung erscheinen lassen. Als stellvertretend für diese Charakterisierung seien zwei Äußerungen Jacob Grimms zitiert, die mitten in der Wörterbuch-Vorrede stehen:

Auch ist gar keine noth, dasz allen alles verständlich (DWB 1, XII).

und wenn es [das Wörterbuch, UHZ] überhaupt nutzen soll, gibt es kein anderes als ein wissenschaftliches. (ebd. XIII)

2 Die Kritik von Rudolf von Raumer 1858/1863 und Hermann Paul 1894 wird hier übersprungen, weil sie wissenschaftlich-methodisch ansetzte; vgl. Kirkness/Kühn/Wiegand 1991, XVII-XXVII.

3 Die Kontroverse zwischen Boehlich und den den DWB-Bearbeitern Th. Kochs und Hans Neumann wird mit Nachweisen referiert in Kirkness/Kühn/Wiegand 1991, XXVIII-XXXI. Vgl. auch Haß-Zumkehr 1995, $383 \mathrm{ff}$.

4 Nachweise in Kirkness/Kühn/Wiegand 1991, XXIX.

5 „und schon ist jenes öffentliche ereignis vor andern noch viel stärker erschütternden, deren vorspiel es gleichsam abgab, in den hintergrund gewichen" (DWB 1, II) - die „Göttinger Sieben“ als Vorspiel der Revolution von 1848, daran will Grimm die vergessliche Öffentlichkeit erinnern.

6 S. Kirkness 1980 sowie die Beiträge, bes. die Einleitung der Herausgeber in dem Sammelband von Kirkness/ Kühn/ Wiegand 1991. 
Den Hintergrund dieser Charakterisierung stellt die Entwicklungsphase der deutschen Philologie um die Jahrhundertmitte dar: Die junge Disziplin hatte einen gewissen Institutionalisierungsgrad erreicht und befand sich in der Professionalisierungsphase, für die ein Ausbau der Methodik durch immer weiter gehende Erprobungen kennzeichnend ist. ${ }^{7}$ Jacob Grimm hatte der historisch-vergleichenden Sprachforschung in seiner Deutschen Grammatik 1819/1822 den Weg gewiesen - 30 Jahre später, im DWB, sollte sie sich in hunderten und tausenden von Einzelwortuntersuchungen bewähren.

Die Widersprüchlichkeit zwischen

a) dem notwendig an „das deutsche Volk“ oder zumindest an eine gesellschaftlich repräsentative soziale Schicht adressierten Nationalwerk und

b) dem Dokument und Experimentierfeld eines im Enstehen begriffenen neuen Wissenschaftsparadigmas und einer neuen wissenschaftlichen Disziplin

ist zwar oft festgestellt, aber bisher unerklärt geblieben. Nachfolgend sollen beide Positionen, zunächst die national-symbolischen Motive und Elemente im Verhältnis zu den nicht-nationalen dargestellt und anschließend der Versuch gemacht werden, den Widerspruch zwischen national-populärer und wissenschaftlicher Tendenz zu erklären.

\section{Die Idee des Nationalwörterbuchs zu Beginn des 19. Jahrhunderts und die Konzeption des Grimmschen Wörterbuchs}

Das herausragende Wörterbuch der deutschen Aufklärung, das Grammatisch-kritische Wörterbuch der hochdeutschen Mundart von Johann Christoph Adelung ${ }^{8}$, war in den Augen der Mit- wie der Nachwelt kein Nationalwörterbuch, so sehr es auch zur Herausbildung der überregionalen deutschen Standard- und Nationalsprache beigetragen hat. Die Idee eines Wörterbuchs, das in einem produktiven Verhältnis zur Nationsbildung steht und das der Sprachgemeinschaft den sinnfälligsten Aspekt von Sprache überhaupt, nämlich ihren Wortschatz, greifbar repräsentiert, entstand als eine von mehreren parallelen Ausprägungen nationaler Symbolik schon während der napoleonischen Herrschaft und entfaltete sich nach dem mi-

Bahner/Neumann 1985; Fohrmann/Voßkamp 1994.

2. verm. u. verb. Ausg. Leipzig 1793-1801. 
litärischen Sieg über Napoleon. Voraussetzung war der Gedanke, dass ein befruchtendes Verhältnis zwischen Sprache und Nation bestünde, dass also die Pflege der Sprache nicht nur diese selbst, sondern auch die Nation bzw. das „Volkstum“ zur „Blüte“ bringen könne, modern gesprochen, dass eine nationale Deutung von Sprache und Wortschatz das Nationalbewusstsein der Deutschen stärken und somit der politischen Nationseinigung den Weg ebnen könnte.

Zur Verknüpfung der beiden Leitideen Sprache und Nation gehörte konstitutiv eine dritte Idee, die die Belebung von Sprache und Nation an die Suche nach beider Vergangenheit und Vorfahren band. Eine ausschließliche Beschäftigung mit gegenwärtiger Sprache und gegenwärtiger Nation war völlig undenkbar; beides war nicht anders als in die historische Perspektive eingebunden, d.h. als Sprach- bzw. Wortgeschichte wahrnehmbar. Das der historischen Perspektive zugrunde liegende Geschichtsverständnis der deutschen Philologie war entwicklungsbezogen und von einer organizistischen Metaphorik geprägt, die das 19. Jahrhundert (nach Goethe) als eine Epoche des Niedergangs gegenüber den Blüteperioden des Alt- und Mittelhochdeutschen darstellte. Die in Archiven und Bibliotheken entdeckten literarischen "Altertümer" mussten dabei die weithin fehlenden nationalgeschichtlichen Realien ersetzen. Wo auch die Textquellen die ersehnten Auskünfte über das Leben der deutschen Vorfahren nicht anschaulich machen konnten, entfalteten sich mehr oder weniger mythologisierende Spekulationen. ${ }^{9}$ Die historische Sprach- und Literaturforschung der Germanistik, zu deren Meilensteinen das Deutsche Wörterbuch zu rechnen ist, war von ihren Anfängen an eine sich national legitimierende Wissenschaft. ${ }^{10}$

Friedrich Ludwig Jahn behandelte 1810 in seinem Buch Deutsches Volksthum den Zusammenhang von Sprachpflege und Nation und kam dabei auch auf die Rolle eines Wörterbuchs zu sprechen. Die Forderung, deutsche Sprachforschung historisch zu betreiben, war bei Jahn mit einem antifranzösischen Affekt verknüpft, denn er setzt die historische Sprachforschung ("grabet die alten Quellen auf“) „Lutetiens stehender Lache“ entgegen, womit das Wörterbuch der Académie Française gemeint ist, das die „edele[n] lebendige[n] Sprache“, den „nieversiegenden Urborn“ durch seinen rationalistisch-normativen Ansatz eingedämmt habe (Jahn o.J.,

9 Berühmte Beispiele liefern die etymologischen Angaben Jacob Grimms in den ersten Bänden des DWB, etwa wenn er zwischen dem Adj. arm und dem Substantiv der Arm (für den Körperteil) einen geschichtlichen Zusammenhang herzustellen versucht: „wie gefühlvoll erschiene die sprache, welcher der arme ein solcher ist, den man mitleidig, liebreich aufnimmt und in die arme schlieszt? arm miser stammte hiernach unmittelbar aus arm brachium, musz nur einen hernach schwindenden ableitungsvocal besessen haben" (DWB 1, 554); vgl. Reichmann 1991, bes. $306 \mathrm{ff}$.

10 Darauf kann hier nicht eingegangen werden, vgl. Bahner/Neumann 1985, Weimar 1989, Fohrmann/Voßkamp 1994. 
128). Die gleiche Gegenüberstellung, wenn auch rational argumentierend, nahm noch Wilhelm Grimm in seinem Bericht über das Deutsche Wörterbuch 1846 vor (W. Grimm 1846, 510-512). Bei Ernst Moritz Arndt tauchte 1818 dann konkret die Vorstellung eines „deutschen Wörterbuchs“ auf, das im Gegensatz zur Académie Française, die „sich bloß hinsetzte und an dem Vorhandenen klaubte, feilte, besserte und regelte“, dasjenige enthielte, was

eine lebendige und frische Gesellschaft, die sich über ganz Deutschland von den Alpen und der Maas und Mosel bis an die Slie und Memel verbreitete und Männer von Kenntnissen und gutem Sinn und Geruch und Fähigkeit, das Lebendige aufzufassen, in die einzelnen Landschaften und Gaue versendete, daß sie aufläsen und erkundeten, was vom Sprachvorrat noch aufzulesen und zu erkunden ist [...] So würden wir besondere Wörterbüchlein der einzelnen Landschaften und Gaue, oft eines einzelnen Tales oder eines Inselchens erhalten; und dann könnte später von geistvollen und gelehrten Männern endlich auch ein deutsches Wörterbuch gefertigt werden. (Arndt o.J. (1. Aufl. 1818), 187)

Wie die Zitate zeigen, wurde die Idee des Nationalwörterbuchs im Gegensatz zum „toten“ Regelwerk der Franzosen als Schatzhaus der lebendigen, im historischen Fluss befindlichen Sprache konzipiert, vor dessen Beginn die Sammelarbeit gesetzt war. 1846 fasste Karl August Varnhagen von Ense zusammen, was die gebildete Welt seiner Zeit mit dem Sammeln von Sprachdenkmälern verband:

Allgemein ist jetzt die Lust am Sammeln, Aufspüren, Bewahren; nie hat man so die Sprachdenkmale der Völker bearbeitet, die Alterthümer überhaupt, die Einzelheiten der Vorzeit, die Eigenthümlichkeiten jedes Orts und jeder Landschaft. Das ist mir grade ein Zeichen, daß in dem nächsten Zeitalter viel untergehen und verschwinden wird, daß es hohe Zeit ist, einige Proben des Frühergewesenen zu erhalten, denn bald wird dieses gänzlich fehlen. Die Brüder Grimm sind solche Retter. Ein ungeheurer Stoff von Gelehrsamkeit drängt sich eilig in sie zusammen, um mit ihnen überzuschiffen. (Varnhagen von Ense, 3. Bd., 1862, 331).

Konkreter als die öffentliche Meinung, die sich in den Stimmen Jahns, Arndts und später Varnhagens äußerte, waren die Nationalwörterbuchideen der Verleger. Drei Verlagshäuser - Cotta in Stuttgart, Karl Reimer von der Weidmannschen Buchhandlung in Leipzig und die Heidelberger Universitätsbuchhandlung Winter - hatten zwischen 1823 und 1838 die Idee zu einem solchen Werk entwickelt und unabhängig voneinander Jacob Grimm angetragen (Kirkness 1980, $54 \mathrm{f}$.). Winter dachte an ein großes mittelhochdeutsches Wörterbuch (ebd. 55), die übrigen eher an ein neuhochdeutsches. Dass die Idee sozusagen in der Luft lag, belegen ferner Äußerungen von Maßmann (ebd. 75) und Müller. Wilhelm Grimm schrieb seinem Bruder am 16. 4. 1838: „Müller [...] sieht die Sache als Nationalwerk an und [...] denkt an 100,000 [Exemplare]“ (ebd. 60).

Die Verleger wussten sich mit ihren Plänen bewegt von der „Zeit gewaltigen nationalen Aufschwungs" (Reimer zit. ebd. 109) und sahen offensichtlich einen Markt für ein großes Wörterbuch, „wie es Deutschland jetzt 
wohl haben sollte“, ohne dass sie zunächst konkrete Vorstellungen über dessen Art und über die Adressatenerwartungen besaßen. ${ }^{11}$ Es war lediglich klar, dass es sich hierbei um eine „literarische Unternehmung“ „von außerordentlicher Bedeutung und großer Wichtigkeit“ handeln würde, die „nicht nur ehrenvoll sondern auch gewinnbringend sein werde“ (Reimer zit. ebd. 53 u. 55).

In dieser Situation gewannen zwei Bedingungen, die das Nationalwörterbuch erfüllen sollte, eine herausragende Bedeutung: der Name Grimm und die Ausrichtung des Werks auf nicht-wissenschaftliche Benutzer. Nicht nur die Verleger, sondern die gesamte sich artikulierende Öffentlichkeit sahen in Jacob und Wilhelm Grimm die prädestinierten Verfasser des Nationalwörterbuchs. Nachdem Reimer die Brüder für seinen Plan gewonnen hatte, rückte die zweite der genannten Bedingungen, die praktisch-populäre Ausrichtung, in den Mittelpunkt der Verlegerinteressen und schuf einen lang andauernden Konflikt zwischen ihm und den Autoren. Reimer war sich mit allen seinen Kollegen darin einig, dass das zeitgemäße Nationalwörterbuch ein populäres sein müsse und kein ausschließlich gelehrtes sein dürfe. Das von der damals größten verlegerischen Autorität Friedrich Perthes 1838 erstellte Gutachten forderte eine klare, wirtschaftlich motivierte Entscheidung für entweder „ein streng wissenschaftlich gehaltnes Wörterbuch“ mit maximal 2000 Exemplaren Auflage oder eines „in Art des Adelungschen“ für „das große Publikum der praktischen Geschäftsmänner, der kaufmännischen Comptoire u.s.w. “ mit größerer Auflage (zit. Kirkness 1980, 62f.).

Reimer bat in den Vertragsverhandlungen immer wieder um „Barmherzigkeit“" gegenüber den „Schwachen, deren Zahl nicht gering sein möchte“, d.h. den Laien (zit. ebd., 118), aber Jacob und Wilhelm Grimm festigten ihre Überzeugung „von der Natur des Wörterbuchs“ immer mehr, dass „der Unterschied zwischen einem gelehrten und einem popularen Werk nicht in der Weise vorhanden seyn wird, wie er [=Reimer, UHZ] ihn annimmt." (W. Grimm zit. ebd. 62). Außerdem vertrauten sie in selbstbewusster Weise der Zugkraft ihres Namens ${ }^{12}$, der manche, von ihren eigenen Vorstellungen abweichenden Erwartungen der Öffentlichkeit kompensieren würde. Jacob und Wilhelm Grimm schickten sich an, die Art und Weise des Nationalwörterbuchs nach eigenen Vorstellungen zu definieren.

11 Die ökonomische Situation im deutschen Buchhandel schien die Absatzchancen eines großen enzyklopädischen Werks zu steigern; so war die Auflagenzahl des Conversationslexicons von Brockhaus von 2000 Exemplaren im Jahr 1809 auf 32000 Exemplare in den Jahren 1818-1823 hochgeschnellt; vgl. Haß-Zumkehr 1995, $286 \mathrm{ff}$.

12 Vgl. Kirkness 1980, 72. Verleger und wissenschaftliche Freunde bestärkten sie darin auch immer wieder, z.B. Reimer: „Bei einem Buche von solchem Namen und solcher Bedeutung, wie Ihr Wörterbuch [...]“ (zit. ebd. 129). 
1840 verwendete zum ersten mal eine amtliche Stelle, der preußische Kultusminister Eichhorn, den Ausdruck "Nationalwerk“ in seiner Einladung an die Brüder, nach Berlin zu übersiedeln (ebd., 104). Entsprechend begegnet der Ausdruck Nationalwerk bei beiden Brüdern zunehmend als Schlagwort mit appellativer Funktion. Von einem Nationalwerk, Nationalwörterbuch bzw. vaterländischen Unternehmen, sogar vom Opfer auf dem Altar des Vaterlands sprachen sie und die ihnen verbundenen wissenschaftlichen Kollegen in der Planungs- und Anfangsphase des DWB immer dann, wenn Subskribenten, Käufer oder Mitarbeiter geworben werden sollten. ${ }^{13}$ In der internen Briefkommunikation der Scientific Community wurde dagegen an keiner Stelle „national" argumentiert; hier besaß die Wissenschaftlichkeit und deren Umsetzung unangefochtene Priorität. Wilhelm Grimm etwa ging auf der ersten Germanistenversammlung 1846 in Frankfurt am Main selbstverständlich von der primär wissenschaftlichen Bedeutung des Wörterbuchs aus, die er für groß genug hielt, eine entsprechend geänderte Erwartungshaltung auch beim nicht wissenschaftlichen Publikum zu erzeugen:

[Die] Wissenschaft suche nicht sich selbst allein, sie sei vorhanden, um den Geist des ganzen Volks (ich begreife alle Stände darunter) zu erheben und auf seinem Wege zu fördern. Möge daher das Wörterbuch nicht bloss die Forschung begünstigen, sondern auch im Stande sein, das Gefühl für das Leben der Sprache zu erfrischen. (W. Grimm $1846,516)$.

Ein anderes Beispiel für diese Haltung war Jacob Grimms Überzeugung, der Einfluss des Wörterbuchs werde ausreichen, quasi im Alleingang eine neue, historisch-etymologisch ausgerichtete Orthografie in Deutschland einzuführen (vgl. Haß-Zumkehr 1997). Der Verleger Hirzel machte sich schließlich zu eigen, was Jacob und Wilhelm Grimm nach außen immer überzeugter vertraten: Dass Wissenschaftlichkeit und Popularität in ökonomisch messbarem Sinn sich nicht nur miteinander vereinbaren lassen, sondern dass letzteres sogar aus ersterem notwendig folge.

Als das Wörterbuch im Mai 1852 schließlich zu erscheinen begann, zeigte sich, dass es in einigen Punkten von der allgemeinen Vorstellung eines Nationalwörterbuchs abwich. Bei den überwiegend begeisterungs- und verehrungsvollen Rezensenten wurden diese Abweichungen als marginal eingestuft und das Werk als Verkörperung des langersehnten Nationalwerks gefeiert. Einige wenige anonyme Rezensenten und die beiden namentlich gekennzeichneten Kritikschriften von Daniel Sanders $(1852,1853)$ und Christian Ludwig Wurm $(1852,1853)$ hielten die Abweichungen jedoch für so gravierend, dass sie dem Ganzen den Status eines für das gesamte Volk oder doch für die gebildeteren Kreise benutzbaren Nachschlagewerks absprachen. Teilweise stimmte die Kritik mit denjenigen Punkten überein, die der Verleger im Briefwechsel mit den Brüdern Grimm angemahnt hatte.

13 Belege ebd., 69, 82, $141 \mathrm{f}$. 
Im Ganzen zeigt sich in den kritischen Äußerungen, dass die Vorstellung des Nationalwörterbuchs in einem Teil der Öffentlichkeit nicht primär historisch-etymologisch, sondern sprachrationalistisch, auf die überregionale und schichtenübergreifende Varietät der Gegenwartssprache und ihre reflexive Beherrschung gerichtet war. ${ }^{14}$ Der Ausdruck national wurde hier mit Bezug auf Sprache und Wörterbuch kaum verwendet; statt dessen war von einem Wörterbuch „für das deutsche Volk“ die Rede:

Die genannte Handlung [die Weber'sche Buchhandlung in Leipzig, UHZ] hatte seit lange den Plan und den Wunsch gehegt, ein solches Werk für das deutsche Volk zu verlegen; aber sie hatte diesen ihren Lieblingsplan von Jahr zu Jahr hinausgeschoben, weil mit Vielen sie von dem lang und vielfach angekündigten Werke der Grimm die hohen Erwartungen theilte, die freilich sehr bald einer schmerzlichen Enttäuschung Platz machten. Mehr als je nun von der Berechtigung und Nothwendigkeit eines neuen Wörterbuchs für das deutsche Volk überzeugt, wandte sie sich an mich. [...] Dass ein neues deutsches Wörterbuch ein Bedürfniss ist, hat das Volk durch die begeisterte Theilnahme anerkannt, mit der es die ersten Hefte des Grimm'schen Werkes aufgenommen. Und wenn dieselbe rasch gesunken ist [...], so ist der Grund davon nicht im Volk, sondern in der Grimm'schen Arbeit zu suchen, die dem, was die Nation erwartete und erwarten darf, in keinerlei Weise genügt, ja der ganzen Anlage nach nicht genügen kann. (Sanders 1854, 3) ${ }^{15}$

Wurm wollte ein Nationalwörterbuch an den Nachschlagebedürfnissen des „praktischen Mannes“ orientieren (Wurm 1852, 33). Wie auch Sanders brachte er in seiner Adressatenbestimmung zum Ausdruck, dass das "Selbstdenken“ jedes „Mitglieds der deutschredenden Gemeinschaft“ im "Geschäfte der Sprachforschung“ als Angelegenheit der gesamten Sprachgemeinschaft gegen den Anspruch gelehrter Experten auf alleinige Zuständigkeit zu verteidigen ist (ebd.).

Wollte Jacob Grimm mit seinem Wörterbuch wie schon mit seiner Deutschen Grammatik die „neue“ Art der Sprachforschung für den Prozess der Herausbildung des Nationalbewusstseins dienstbar machen, so ist für die Generation der „kritischen Enkel“ Sanders und Wurm die Nationalsprache bereits potenzieller Besitz, den es allerdings den durch Demokratisierung, Industrialisierung und Überregionalisierung veränderten Kommunikationsbedürfnissen gemäß auszubauen gilt.

Beide Kritiker haben den Versuch unternommen, dem Grimmschen Werk ein eigenes Nationalwörterbuch entgegenzusetzen; während Wurm (1858) nur einen von mehreren geplanten Bänden herausbringen konnte, gelang Sanders die Publikation eines großformatigen Dreibänders inner-

14 Für Sanders sind diese Vorstellungen ausführlich dargelegt in Haß-Zumkehr 1995, bes. Kapitel 3 und 4; zu Wurm ebd. 283-298.

15 Später war realistischer von einem Wörterbuch „für den weiten Kreis aller gebildeten Deutschen“" (Sanders 1859, s. Haß-Zumkehr 1995, 409 Anm. 165) und für „alle gebildeten oder doch nach Bildung strebenden Schichten unseres Volkes“ (Sanders 1892, s. Haß-Zumkehr 1995, 410 Anm. 167) die Rede. 
halb von 6 Jahren (Sanders 1860/1969) und eines Ergänzungsbandes 20 Jahre später. Die Idee des Nationalwörterbuchs wurde also zu Beginn des 19. Jahrhunderts im gebildeten Bürgertum geboren, und dann von der um Etablierung bemühten neuen germanistischen Wissenschaft aufgegriffen, die ihre gesellschaftliche Legitimation durch Berufung auf ihre nationalen Leistungen, insbesondere durch das Deutsche Wörterbuch, stärken wollte, aber ihr wissenschaftliches Selbstverständnis vor allem aus dem neuen $\mathrm{Pa}$ radigma der historisch-etymologischen Methode bezog. Der nicht nur wissenschaftliche, sondern auch gesellschaftlich herausragende Ruhm der Brüder Jacob und Wilhelm Grimm trug entscheidend zur Durchsetzung ihrer Vorstellung vom Nationalwörterbuch bei, so dass die sich artikulierenden Alternativen bis in die Gegenwart kaum zur Kenntnis genommen wurden.

\section{National-symbolische Elemente des DWB in 108jähriger Entstehungszeit}

Die nachfolgenden Erläuterungen ziehen jeweils alle vier Bearbeitungsphasen des DWB zwischen 1852 und 1960 in Betracht, die von zum Teil erheblich voneinander abweichenden Vorgehensweisen sowie von Korrekturen bestimmt wurden, ohne eine gewisse Übereinstimmung mit der Grimmschen Konzeption völlig aufzugeben. ${ }^{16}$

\subsection{Die Quellen- und Stichwortwahl}

Das Werk sollte zunächst drei, dann vier Jahrhunderte, von Luther bzw. von der Mitte des 15. Jahrhunderts bis Goethe bzw. bis zur jeweiligen Bearbeitungszeit umfassen und dabei alle Stilschichten und alle Mundarten berücksichtigen. Das entscheidende Kriterium für die Quellen- und für die Stichwortauswahl war für Jacob und Wilhelm Grimm die „sprachgewalt“ eines Dichters, seine „kräftige ausdrucksweise“ (DWB 1, XVII) oder die „lebendigkeit der redensarten“ (ebd. XVIII). Fachsprachliche Quellen sowie Neubildungen barocker oder klassischer deutscher Herkunft kamen ihnen daher oft saft- und kraftlos vor. Schiller etwa, so Jacob Grimm in einem Brief an Karl Lachmann 1838, sei „wortarm und unsrer sprache nicht recht mächtig" gewesen (zit. Kirkness 1980, 70). Das Volkstümliche, Einfache der Literatur rangierte in Grimms Augen weit höher als das Verfeinerte oder Höfische und auch höher als das Bürgerlich-Moderne:

16 Zur Charakterisierung der vier Phasen siehe Bahr 1991. 
Bei der Quellenauswahl legten die Brüder Grimm einen besonderen Akzent auf das Historisch-Dynamische, auf das Volksmäßige und das Naiv-Poetische. Sie bevorzugten eine natürliche, lebendige, konkret-anschauliche, kraftvoll-derbe Ausdrucksweise, wie sie auch bei mittelmäßigen Schriftstellern am ehesten in älteren volksnahen Quellen anzutreffen war. Wie sie wiederholt hervorhoben, suchten sie hauptsächlich nach heimischen Simplizia und Redensarten, auch und insbesondere den seltenen und veralteten, sowie nach unbekannten, ungewöhnlichen oder vom gegenwärtigen Gebrauch abweichenden Bedeutungen, vor allem von einfachen Wörtern. Provinzialismen und Ableitungen interessierten weniger, während frei auflösbare Komposita und Fremdwörter nicht gefragt waren. (ebd., 15).

Dies blieb die Richtschnur auch für die späteren Bearbeitungsphasen, obwohl in ihr inakzeptable Beschränkungen lagen, die wenigstens punktuell aufgehoben werden mussten. Dies änderte aber nichts daran, dass mit dem wissenschaftlich begründeten Desinteresse am Lehnwortschatz und an den lexikalischen Folgen produktiver Wortbildungsmittel und -verfahren die Inhalte oder Diskurswelten ganzer literarischer Bereiche ausgeblendet wurden: Politik und Gesellschaft (Holly 1991), Handel, Manufakturwesen, ein großer Teil der Wissenschaften und die Technologie (Schiewe 1991) mit Ausnahme des altständischen Handwerks in Pressetexten und Literatur. Die Konzentration auf das Volkstümlich-Derbe, für das Luther das prototypische Beispiel abgab, brachte eine Vernachlässigung unter anderem der lange Zeit lateinisch dominierten katholischen Literatur mit sich, was schon 1852 als ideologische Einseitigkeit kritisiert wurde (Haß-Zumkehr 1997).

In späteren Bearbeitungsphasen, besonders zu Beginn der 3. Phase ab 1908, musste die Quellenbasis um ein Mehrfaches verbreitert und die Arbeit insgesamt reorganisiert werden (Bahr 1991, $29 \mathrm{ff}$.). Die Charakterisierung der nach und nach neu hinzu gekommenen Quellen und ihre literaturgeschichtliche Einordnung ist noch Desiderat (Kirkness/Kühn/Wiegand 1991, XXXVI); deshalb kann hier nur als Gesamteindruck geäußert werden, dass das Grimmsche Basiskriterium der „Sprachgewalt“ eines für das Wörterbuch auszuwertenden Textes seine Rolle bis zum Schluss der Bearbeitungszeit beibehielt. Sicherlich ist aber als pragmatisches Auswahlkriterium die vom allgemeinen Bildungswissen der leitenden Lexikographen aus beurteilte "Wichtigkeit“ eines Autors bzw. eines Textes hinzugekommen. Dieses Bildungswissen war für die gesamte Bearbeitungszeit trotz gewisser Einschränkungen in der nationalsozialistischen Zeit humanistisch geprägt. In diesen Gesamteindruck hinein gehören auch die quantitativ sicher marginalen Quellen nationalistischer und nationalsozialistischer Provenienz ${ }^{17}$, aber sie haben in der bundesrepublikanischen Öf-

17 Die im „Dritten Reich“ entstandenen Bände des DWB, das Ende von G und große Teile von S, T und U, lemmatisieren ein paar NS-Neuwörter, etwa Sturmabteilung mit Belegen von Hitler und Horst Wessel. 
fentlichkeit nach 1945 und vor dem Hintergrund des völkischen Nationalismus eine symbolisch-heraushebende Deutung erfahren. ${ }^{18}$ Ebenso marginal im qualitativen Sinn sind die Quellen und besonderen Wortbedeutungen aus der Presse der DDR, ohne dass ihnen eine vergleichbare Aufmerksamkeit widerfahren wäre. ${ }^{19}$

Das Bild von Nationalsprache, das im DWB durch die Quellen- und Stichwortwahl im Großen und Ganzen vermittelt wurde, entspricht nicht genau der Varietät, die die Soziolinguistik als deutsche Nationalsprache des späten 19. und der ersten Hälfte des 20. Jahrhunderts beschreiben würde. Es ist obendrein ein relativ vages und affektiv geprägtes Bild, das seine Verwandtschaft mit der barocken Idee des „kerndeutschen Stammwortschatzes“ (vgl. Gardt 1994) nicht zu verleugnen vermag, aus dem alle entlehnten und alle nur abgeleiteten oder zusammengesetzten Elemente ausgeschlossen sind und der eine den germanischen Vorfahren zugeschriebene Weltsicht zu repräsentieren oder wenigstens näherzubringen scheint.

\subsection{Belegpräsentation}

Die Schatzhaus- und Füllhorn-Metaphorik (s.o.) bezieht sich auf die Art und Weise, mit der die benutzten Quellen in die Belegteile der Wortartikel Eingang gefunden haben. Das Kriterium der „Sprachgewalt“ verlangte eine umfassend veranschaulichende Darstellung, keine analytisch-abstrahierende Exemplifizierung. Das DWB sah sich von Anfang an und zunehmend mit dem Problem einer unkontrollierten Umfangserweiterung konfrontiert, die ausschließlich auf die Menge der zitierten Belege zurückzuführen ist. Nicht im Hinblick auf den lemmatisierten Wortschatz, wohl aber im Hinblick auf die literarische Dokumentation der ausgewählten Stichwörter erfüllt das DWB eine Thesaurus-Funktion. Die Chance, mit Hilfe des DWB ein bekanntes Zitat unbekannter Herkunft zu identifizieren und es quasi als „gehobenen Büchmann" benutzen zu können, ist relativ groß.

Eine Funktionsanalyse der Beispiele und Belege im DWB (Haß 1991) hat ergeben, dass zwei von fünf möglichen, weil tradierten Funktionen vorherrschend waren: An die Grimmsche Idee von der „Sprachgewalt, die den Deutschen vor Augen zu stellen " ist, schloss sich unmittelbar eine ostensive Funktion an, ein unausgesprochenes „Seht her und staunt!“. Ab der zweiten, besonders ab der dritten Bearbeitungsphase dominierte hingegen die philologische Nachweisfunktion, bei der die Materialdarstellung die wissenschaftlichen Aussagen absichert. Beide Funktionen werden durch Quantität verstärkt.

18 Boehlich 1952, Boehlich 1960, Boehlich 1961, Jens 1984.

19 Vgl. v. a. die Wortartikel Westen und westlich in DWB 29 (1960). 
Durch die äußerst umfangreiche Belegdarstellung rückt die ausgewählte Literatur selbst ins Zentrum des DWB, das man so gesehen als eine alphabetisch zerstückelte National-Anthologie begreifen könnte. Das DWB-spezifische Bild der Nationalsprache wurde als Literatursprache festgelegt, in die nur Bruchstücke von Gebrauchssprache einbezogen sind. Welcher literarische Kanon hierdurch repräsentiert wird, ist noch nicht untersucht (s.o.).

\subsection{Vorrang von Etymologie und Formgeschichte vor Semantik}

Jacob Grimms bahnbrechenden Erkenntnisse über den Lautwandel hatten eine Etymologie im wissenschaftlichen Sinne überhaupt erst möglich gemacht, die somit das methodische Herzstück des neuen Wissenschaftsparadigmas ausmachte. Hinzu kam dessen Innovations- und Korrekturbewusstsein gegenüber der rationalistischen Sprachreflexion des 18. Jahrhunderts, in deren Lexikographie die Herausarbeitung von Wortbedeutungen, Bedeutungsgrenzen und Synonymendifferenzierungen eine große, hier nicht zu erläuternde Rolle spielte. „Der volle Gehalt [der Wörter, UHZ] lässt sich durch keine Definition erklären“, verkündete Wilhelm Grimm $(1846,513)$ in seinem Bericht über das Deutsche Wörterbuch, dem eigentlich programmatischen Text des DWB ${ }^{20}$; statt dessen solle es um die „Naturgeschichte der einzelnen Wörter" gehen (ebd.). Folgerichtig dienten den Brüdern überwiegend nur knappe lateinische Äquivalente als Bedeutungsangaben. Der „Urbegriff" (Reichmann 1991) hingegen ist eine in den Wortursprung verlegte Größe, die Einheit und Kontinuität der Naturgeschichte des Worts sichert und die sich nur in den Quellen selbst spekulativ aufspüren, nicht analytisch absondern und explizieren lässt.

War bei den Brüdern Grimm die Etymologie noch eine Verbindung aus Formgeschichte und Urbegriffsentwicklung gewesen, so wurde sie bei den nachfolgenden Bearbeitern zu einer rein ausdrucksseitigen Angelegenheit, denn eine sprachtheoretische Fixierung des „Urbegriffs“ als einer semantischen Größe hatte Grimm nicht hinterlassen. Das Primat der Etymologie vor jeder Semantik trug in das vom DWB vermittelte Bild der Nationalsprache ein Element ein, das Sprach- bzw. Wortgeschichte mit Etymologie gleichsetzte.

20 Zu dieser Einstufung Haß-Zumkehr 1997. 


\section{Nicht-nationale Merkmale des Deutschen Wörterbuchs}

Es waren vor allem zwei Merkmale, die von den ersten öffentlichen Reaktionen an (vgl. Haß-Zumkehr 1997) als Widerspruch gegen die angeblich angestrebte Popularität des Wörterbuchs markiert wurden: die vor allem in den ersten beiden Bearbeitungsphasen regelmäßige Zuhilfenahme lateinischer Äquivalente bei den Bedeutungsangaben, z. B. „einwohnend, inhabitans, immanens" (DWB 3, 345), und die Entscheidung für die Antiqua- anstelle der seinerzeit üblichen „deutschen“ Fraktur-Schrift. ${ }^{21}$

Die lateinischen Äquivalente entsprachen der älteren, voradelungischen lexikographischen Tradition und spiegeln vor allem das Desinteresse an einer differenzierten Semantik; die lateinischen Äquivalente sollten im DWB nichts weiter als die ungefähre Zuordnung der den diversen germanischen und sonstigen Sprachen entstammenden Wortformen zu einer Inhaltseinheit und damit ihre Identifizierung und Abgrenzung von Homonymen ermöglichen. Die umfangreichen etymologischen „Köpfe“ der Wortartikel des DWB sind sprachvergleichende Abhandlungen, in denen mittels der lateinischen Angaben das tertium comparationis fixiert wurde. In den späteren Bearbeitungsphasen wurden die lateinischen Äquivalente durch neuhochdeutsche ersetzt oder ganz zugunsten formgeschichtlicher Erläuterungen aufgegeben. Das wichtigste war und blieb die historische Sprachvergleichung, eine in dieser Darstellugsform gänzlich unpopuläre und ausschließlich wissenschaftliche Texthandlung. Die Verwendung lateinischer Äquivalente signalisierte die nie infrage stehende Wissenschaftlichkeit nur besonders stark und war von Sanders und Wurm als Gestus distanzierender „Gelahrtheit" entsprechend kritisiert worden.

Eine mindestens ebenso auffällige Abweichung von den Publikumserwartungen stellten Schrift und Schreibung dar. In seiner Wörterbuch-Vorrede bewertete Jacob Grimm die Fraktur als „verdorben“ und „geschmacklos“, als „eckig, knorrig und scharf“ (DWB 1, LII) und begründete die Wahl der Antiqua als die unverdorbene und ältere: „jeder kundige weisz, dasz im mittelalter durch das ganze Europa nur éine schrift, nemlich die lateinische für alle sprachen galt und gebraucht wurde“ (ebd.). Im Übrigen sei auch die „vulgarschrift“, d.i. die Fraktur, keineswegs ausschließlich für das Deutsche in Gebrauch, sondern für eine Reihe weiterer nord- und osteuropäischer Sprachen, und könne damit keineswegs „deutsch“ genannt werden (ebd. LIII). Grimm führte dann sieben Argumente gegen die Fraktur an, von denen sechs orthografischer Natur sind. Für Grimm hat die Fraktur die Entwicklung der deutschen Rechtschreibung in Vielem ungünstig beeinflusst, z.B. hat sie „den albernen gebrauch grozser buchstaben für alle substantive

21 Zur nationalsymbolischen Bewertung der Schriftarten vgl. von Polenz 1996. 
[veranlaszt]“, und „sie kann den unterschied der majuskel I und J nicht ausdrücken, und musz für beide J verwenden, auch entgehn ihr die accente“ (ebd.). Das letzte Argument ist entschieden ökonomisch und internationalistisch: „sie hindert die verbreitung deutscher bücher ins ausland, und ist allem fremden widerwärtig" (ebd.).

Grimms Vorstellung einer mit Hilfe des DWB zu reformierenden deutschen Orthografie war also nicht nur national-historisch motiviert, insofern er veraltete Schreibungen favorisierte, sondern entstammt dem seit dem Mittelalter tradierten Horizont der gesamteuropäischen Scientific Community, der Grimm sich zugehörig fühlte.

Insgesamt sind es also drei Merkmale, die die nationalsymbolische Funktion des DWB in der Öffentlichkeit eher abschwächten: die durch das Latein markierte Gelehrsamkeit, die Antiqua und die „europäische“ Tendenz in den Grimmschen Bestrebungen einer Orthografiereform. Alle drei Merkmale finden ihre Erklärung in der wissenschaftlichen Ausrichtung des Wörterbuchkonzepts, die die Brüder Grimm und auch die späteren Bearbeiter niemals zugunsten gesellschaftlicher Erwartungen aufgaben. Im Unterschied zu diesen drei Merkmalen lag bei den unter Punkt 3 erläuterten nationalsymbolischen Merkmalen eine Koinzidenz von gesellschaftlichen Erwartungen und wissenschaftlichen Zielen der historischen Sprachforschung vor.

\section{Resümee}

Zusammenfassend lässt sich feststellen, dass die nationale Funktion des DWB von den germanistischen Lexikographen bewusst nur gegenüber der Öffentlichkeit, also in fachexterner Adressierung, zum Ausdruck gebracht wurde, während alle fachinternen, an die Angehörigen der Scientific Community adressierten Äußerungen über die Aufgaben des Wörterbuchs und vor allem die Wörterbuchpraxis selbst den wissenschaftlichen Fortschritt und die Etablierung des neuen Paradigmas der deutschen Philologie als konstitutiv historischer Sprachforschung hervorheben. Mit dieser Erklärung erhielte die nationale Symbolik des DWB, pointiert gesagt, das Aussehen eines gezielten Schachzuges von Wissenschaftlern, die ihr Fach in der Phase der institutionellen Verfestigung auch der gesellschaftlichen Anerkennung zu versichern trachteten und dabei den nationalen, in diesem deutschen Falle speziell national-kulturellen „Geist“ der Zeit zwischen 1848 und 1871 zu nutzen wussten.

Diese sehr zugespitzte Formulierung scheint jedoch einen Gegensatz zwischen ideologischem und wissenschaftlichem Handeln vorauszusetzen. Allerdings muss man fragen, inwiefern sich das wissenschaftliche Para- 
digma der Germanistik selbst als national begründet verstand. Der bis mindestens um 1900 ausschließlich historische Blick der Germanistik ist fast schon gleichzusetzen mit nationaler Orientierung, weil alle nationalen Bewegungen in der deutschen Geschichte (vom Humanismus an) bei den vermeintlichen Traditionen und Vorfahren in Sprache, Literatur, Mythos, Recht und Religion ansetzten. Die Leitideen Nation bzw. Volk und Geschichte, letztere verstanden als Überlieferung der Lebensäußerungen der Vorfahren, sind zumindest in deutschen Verhältnissen enger aufeinander bezogen und aufeinander projiziert als mit den weiteren Leitideen (Kultur, Sprache, Recht, Religion).

Jacob Grimms fast beschwörende Anknüpfung an die Göttinger Sieben in seiner Vorrede zum DWB sowie die in seinen Schriften mehr metaphorisch eingeübte als theoretisch fundierte Verbindung von Sprache und Volk auf der einen Seite und die adressatenbewusste Wahl nationaler Appelle und wissenschaftlicher Argumentationen auf der anderen Seite lassen den Schluss zu, dass die germanistische Wissenschaft sich ihres anfänglichen Gleichklangs und ihrer übereinstimmenden Motive mit den politisch-nationalen Bestrebungen vom Anfang des 19. Jahrhunderts bewusst war, diese anfänglichen und deshalb grundsätzlichen Übereinstimmungen auch im öffentlichen Bewusstsein gewahrt wissen wollte, dass sie den Prozess fortschreitender Institutionalisierung und Professionalisierung der Disziplin aber durch keinerlei populäre Anpassungen einzuschränken gedachte, auch nicht im „Nationalwörterbuch“ DWB.

An der national-symbolischen Funktion des Werks hat sein gelehrsames Image und auch die Konkurrenz des für Laien zugänglicheren und sprachaufklärerischen Nationalwörterbuchs von Daniel Sanders nichts geändert. Die Geschichtsschreibung der Germanistik hat sich bis in die 80er Jahre des 20. Jahrhunderts an die Perspektive der Brüder Grimm gebunden und alle nicht programmatisch historischen Alternativen in der Lexikographie als unwissenschaftlich verdrängt.

\section{Literatur}

\subsection{Quellen}

Arndt, Ernst Moritz, Werke. Auswahl in zwölf Teilen. Hrsg. v. August Leffson u. Wilhelm Steffens. 9. Teil (= Geist der Zeit IV). Berlin usw. o.J. (1912).

Boehlich, Walter, Säkularfeier oder Säkulartrauer? Das Deutsche Wörterbuch der Brüder Grimm. In: Merkur 6, 1952, 779-786.

-, Ein Pyrrhussieg der Germanistik. In: Der Monat 13, 1960-61, Heft 154, 38-53. 
-, Blick zurück im Grimm. In: Der Monat 14, 1961-62, Heft 159, 80-85.

Deutsches Wörterbuch von Jacob Grimm und Wilhelm Grimm. 33 Bde. Leipzig, Berlin 1852-1971. Fotomechanischer Nachdruck München 1984.

Grimm, Jacob, Vorrede. In: Jacob Grimm und Wilhelm Grimm, Deutsches Wörterbuch. Bd. 1. Leipzig 1854, Sp. I-LXVIII (Nachdruck München 1984).

Grimm, Wilhelm, Bericht über das Deutsche Wörterbuch (1846). In: Wilhelm Grimm. Kleinere Schriften 1 (1881). Nach d. Ausg. von Gustav Hinrichs neu hrsg. v. Otfrid Ehrismann. Hildesheim, Zürich, New York 1992 (= Jacob und Wilhelm Grimm Werke. Abt. II, Bd. 31), 508-520.

Jahn, Friedrich Ludwig, Deutsches Volkstum. Hrsg. v. Franz Brümmer, Leipzig (Reclam) o.J. (1. Aufl. Lübeck 1810).

Jens, Walter, Das Vorratshaus der Deutschen. Zur Geschichte und Bedeutung des grimmschen Wörterbuchs. München 1984.

Sanders, Daniel, Programm eines neuen Wörterbuches der deutschen Sprache. Leipzig 1854.

-, Das deutsche Wörterbuch von Jacob Grimm und Wilhelm Grimm kritisch beleuchtet. Hamburg. Heft 1, 1852, 104 S. - Heft 2, 1853, 232 S.

-, Wörterbuch der deutschen Sprache. Mit Belegen von Luther bis auf die Gegenwart. 1. Aufl. Leipzig. Bd. 1: 1860, Bd.2, 1. Hälfte: 1863. Bd.2, 2. Hälfte: 1865. Reprograph. Nachdr. d. Ausg. 1876 mit e. Einf. u. Bibliographie v. Werner Betz. Hildesheim 1969.

Varnhagen von Ense, Karl August, Tagebücher aus dem Nachlaß, 1.-11. Bd., Leipzig 1861-1869.

Wurm, Christian Friedrich Ludwig, Zur Beurtheilung des deutschen Wörterbuches von Jakob und Wilhelm Grimm, zugleich ein Beitrag zur deutschen Lexikographie. München 1852.

-, Beleuchtung der Anzeige der fünften Lieferung des deutschen Wörterbuches von Jakob und Wilhelm Grimm. Ein neuer Beitrag zur Beurtheilung desselben. München 1853.

-, Wörterbuch der deutschen Sprache von der Druckerfindung bis zum heutigen Tage, 1. Band. Freiburg 1858 (mehr nicht erschienen).

\subsection{Forschungsliteratur}

Bahr, Joachim, Periodik der Wörterbuchbearbeitung: Veränderungen von Wörterbuchkonzeptionen und -praxis. In: Kirkness, Kühn u. Wiegand (Hrsg.), 1. Teilbd., Tübingen 1991, 1-50.

Fohrmann, Jürgen u. Wilhelm Voßkamp (Hrsg.), Wissenschaftsgeschichte der Germanistik im 19. Jahrhundert. Stuttgart 1994.

Gardt, Andreas, Sprachreflexion in Barock und Frühaufklärung. Entwürfe von Böhme bis Leibniz. Berlin 1994.

$\mathrm{Ha}$, Ulrike, Zu Bedeutung und Funktion von Belegen und Beispielen im Deutschen Wörterbuch von Jacob Grimm und Wilhelm Grimm. In: Kirkness, Kühn u. Wiegand (Hrsg.), 2. Teilbd., Tübingen 1991, 535-594.

Haß-Zumkehr, Ulrike, Daniel Sanders. Aufgeklärte Germanistik im 19. Jahrhundert. Berlin 1995.

-, „alle welt erwartet hier eine erklärung von mir“ - Jacob Grimms Vorrede zum Deutschen Wörterbuch zwischen Apologie und Programm. In: Zeitschrift für germanistische Linguistik 25, 1997.

Holly, Werner, „Wilde pflanzen ohne nährende frucht“ - Die Behandlung des politischsozialen Wortschatzes im Deutschen Wörterbuch. In: Kirkness, Kühn u. Wiegand (Hrsg.), 2. Teilbd., Tübingen 1991, 347-405. 
Kirkness, Alan, Geschichte des Deutschen Wörterbuchs 1838-1863. Stuttgart 1980.

-, Peter Kühn u. Herbert Ernst Wiegand (Hrsg.), Studien zum Deutschen Wörterbuch von Jacob Grimm und W ilhelm Grimm. 2 Teilbände. Tübingen 1991.

Polenz, Peter von, Die Ideologisierung der Schriftarten in Deutschland im 19. und 20. Jahrhundert. In: Karin Böke, Matthias Jung u. Martin Wengeler (Hrsg.), Öffentlicher Sprachgebrauch. Praktische, theoretische und historische Perspektiven. Georg Stötzel zum 60. Geburtstag gewidmet. Opladen 1996, 271-282.

Reichmann, Oskar, Zum Urbegriff in den Bedeutungserläuterungen Jacob Grimms (auch im Unterschied zur Bedeutungsdefinition bei Daniel Sanders). In: Kirkness, Kühn u. Wiegand (Hrsg.), 1. Teilbd., Tübingen 1991, 299-345.

Schiewe, Jürgen, Fach- und Wissenschaftssprachen im Deutschen Wörterbuch. In: Kirkness, Kühn u. Wiegand (Hrsg.), 1. Teilbd., Tübingen 1991, 225-263.

Schmidt, Hartmut, Deutsches Wörterbuch von Jacob Grimm und Wilhelm Grimm,... Sonderauflage anläßlich des 200. Geburtstages ... In: Zs. für Phonetik, Sprachwissenschaft und Kommunikationsforschung 39, 1986, H.6, 712-716.

Sellin, Volker, Nationalbewußtsein und Partikularismus in Deutschland im 19. Jahrhundert. In: Jan Assmann u. Tonio Hölscher (Hrsg.), Kultur und Gedächtnis. Frankfurt/M. 1988, 241-264. 